\title{
DIMENSÃO TERRITORIAL E INOVAÇÃO: UMA APROXIMAÇÃO ENTRE ECONOMIA E GEOGRAFIA
}

\author{
Mateus Boldrine Abrita \\ Professor efetivo na Universidade Estadual de Mato Grosso do Sul (UEMS) \\ Universidade Estadual de Maringá (PCE/UEM) \\ mateusabrita@hotmail.com
}

Rafaella Stradiotto Vignandi

Professora Colaboradora Adjunta A do Departamento de Economia (DCO) Universidade Estadual de Maringá (UEM)

Marcos Paulo da Silva Falleiro

Doutorando em Economia do Desenvolvimento Universidade Federal do Rio Grande do Sul (UFRGS)

\begin{abstract}
RESUMO
O objetivo principal deste artigo foi construir um debate das principais teorias que cercam a geografia, a inovação e o desenvolvimento econômico sob uma perspectiva regional. O ponto central da discussão será como a proximidade geográfica é a chave para a produção efetiva e transmissão partilhada do conhecimento tácito, e como ela reforça a importância dos aglomerados inovadores, dos distritos e das regiões. Para tanto, os esforços foram no sentido de discutir as relações entre inovação, capitalismo e desenvolvimento econômico. Posteriormente, abordou-se as questões de inovações e estrutura produtiva. $E$, na sequência, proporcionou-se uma discussão a respeito das firmas, do aprendizado e da relevância da dimensão territorial. Um ponto essencial que surgiu no debate é justamente a inserção da dimensão territorial na análise. Essa inserção ocorre, dentre outros motivos, pela existência e importância do conhecimento tácito no processo inovador.
\end{abstract}

Palavras-chave: Geografia. Inovação. Desenvolvimento. Economia. Região.

\section{TERRITORIAL DIMENSION AND INNOVATION: APPROXIMATION BETWEEN ECONOMY AND GEOGRAPHY}

\begin{abstract}
The main purpose of this article was to conduct a debate of the main theories surrounding geography, innovation and economic development from a regional perspective. The focal of the discussion will be how the geographical proximity is the key to the effective production and shared transmission of tacit knowledge, and how it reinforces the importance of innovative clusters, districts and regions. Therefore, the efforts were to discuss the relations between innovation, capitalism and economic development. Subsequently, we discussed the issues of innovations and productive structure. And, in the sequence, we discussed about the firms, the learning and the relevance of the territorial dimension. An essential point that arose in the debate is precisely the insertion of the territorial dimension in the analysis. This insertion occurs, among other reasons, because of the existence and importance of tacit knowledge in the innovative process.
\end{abstract}

Keywords: Geography. Innovation. Development. Economics. Region.

\section{INTRODUÇÃO}

A geografia é muito importante na compreensão do complexo processo de geração de conhecimento e inovação. O conhecimento, o processo inovativo e o desenvolvimento econômico são motivados e reproduzidos de forma desigual, principalmente no que diz respeito ao espaço local, regional e temporal. Asheim e Gertler (2006) argumentam que a inovação não é distribuída aleatoriamente ou uniformemente no espaço e que existe uma tendência à concentração espacial, processo que tem se intensificado no decorrer do tempo.

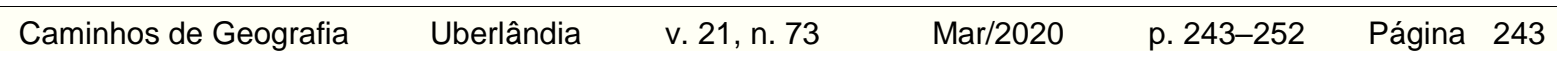


De um modo geral, quanto mais intensiva em conhecimento a atividade econômica for mais concentrada geograficamente tende a ser e pode inclusive ser constatado por investigações empíricas como Gotardo e Staduto (2016). Essa realidade contradiz as previsões de que o uso crescente de informações e tecnologias levaria à dispersão da atividade inovadora. A contemporaneidade econômica é caracterizada por alta competitividade, nos quais o sucesso depende cada vez mais da capacidade de produzir produtos e processos novos ou melhorados. Um efeito da globalização em curso é que muitas capacidades e fatores de produção previamente localizados se tornaram onipresentes. A inovação passou a ser baseada cada vez mais sobre as interações e os fluxos do conhecimento entre os agentes econômicos, como empresas clientes, fornecedores, concorrentes, organizações de pesquisa, universidades, instituições de pesquisa públicas e privadas e agências públicas de tecnologia, centros de transferência e agências de desenvolvimento.

Nesse contexto, o propósito deste artigo será de construir um debate das principais teorias que cercam a geografia, a inovação e o desenvolvimento econômico sob uma perspectiva regional. $O$ tema de destaque será como a proximidade geográfica é a chave para a produção efetiva e transmissão partilhada do conhecimento tácito, e como ela reforça a importância dos aglomerados inovadores, dos distritos e das regiões. Assim, na próxima seção serão discutidas as relações entre inovação, capitalismo e desenvolvimento econômico. Posteriormente, serão abordadas as questões de inovações e estrutura produtiva. Na sequencia, ocorrerá uma discussão a respeito das firmas, do aprendizado e da relevância da dimensão territorial. Por fim, algumas considerações finais.

\section{INOVAÇÃO, CAPITALISMO E DESENVOLVIMENTO ECONÔMICO}

Uma mudança qualitativa de transformação da estrutura produtiva de determinada região pode estar estreitamente relacionada com o desenvolvimento econômico deste local. Schumpeter (1982/1911), através de seu conceito de destruição criadora, demonstra a importância fundamental da mudança tecnológica no processo de desenvolvimento de uma economia capitalista. Nesse sentido, orientar a economia para setores que geram externalidades e efeito de transbordamentos positivo, de empregos de alta qualidade e que elevam a produtividade, são elementos fundamentais para que ocorra o desenvolvimento econômico.

Schumpeter (1982/1911) observa que o sistema capitalista apresenta, em sua própria natureza, uma forma ou método de mudanças econômicas que não podem cessar nunca. Esse caráter evolutivo do processo capitalista não se deve meramente ao fato de a vida econômica acontecer num ambiente social em mutação, tampouco a um aumento quase automático da população e do capital. $\mathrm{O}$ impulso fundamental que inicia e mantém o movimento da máquina capitalista decorre de novos bens de consumo, novos métodos de produção ou transporte, novos mercados, e novas formas de organização industrial que a empresa capitalista gera.

Schumpeter foi o pioneiro em uma escola de pensamento que leva seu nome. Possas (1989) afirma que existe uma corrente econômica mais atual que usa os escritos de J. Schumpeter como inspiração e que poderíamos chamá-los de Neo-Schumpeterianos, apesar de a terminologia ainda não estar consagrada na literatura. Nas palavras de Possas (1989, p. 158), essa corrente de pensamento procura "focalizar o processo de transformação econômica e institucional que periodicamente tem lugar nas economias capitalistas, em diferentes graus de intensidade e abrangência, sob o impacto das inovações tecnológicas".

Uma das abordagens que se enquadraria nessa corrente neo-schumpeteriana é a abordagem evolucionista de Nelson e Winter (1982). A ideia evolucionista traz uma analogia com a biologia. Dessa forma, da mesma maneira como a evolução das espécies acontece por meio de mutações genéticas que deverão passar pelo crivo da seleção do meio ambiente, as mudanças econômicas, representadas pelas inovações, seriam submetidas aos mecanismos de seleção do mercado e da concorrência. Nesse sentido, a teoria evolucionista faz uma análise da mudança tecnologia, principalmente dentro da firma, através da ideia de rotina de comportamento e de tomada de decisões no interior das empresas e instituições. A partir das rotinas existentes e da sua mudança, Nelson e Winter (1982) mostram como acontece à busca (search) das empresas por novas oportunidades de inovação e como o ambiente competitivo e de mercado atuam na seleção (selection) dessas inovações. A grande contribuição da abordagem evolucionista, segundo Possas (1989), é sua visão do processo de geração e difusão da inovação como sendo algo endógeno à estrutura produtiva da indústria e sua análise consistente da dinâmica produtiva da indústria.

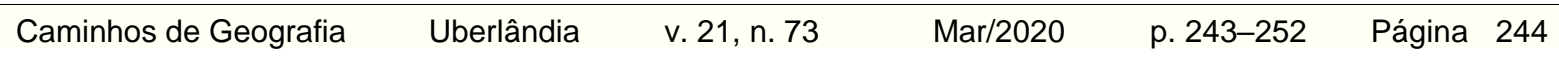


Para Freeman (1995), que se ateve a uma pesquisa mais empírica e menos teórica, por outro lado, foi na Alemanha do século 19 que ocorreu a maior inovação institucional: os departamentos industriais internos de Pesquisa e Desenvolvimento (P\&D). A indústria de corantes da Alemanha percebeu que poderia ser lucrável organizar a pesquisa para novos produtos e o desenvolvimento de novos processos químicos de uma forma regular, sistemática e profissional. Segundo Freeman (1995), foi esse processo que permitiu que a indústria química alemã fosse líder mundial antes e após a Primeira Guerra Mundial. Além disso, após estudos sobre P\&D industrial no Japão, EUA e Europa, argumentou que o ritmo de difusão e os ganhos de produtividade associados dependiam de uma ampla variedade de influências além da P\&D formal: Inovações incrementais seriam produtos de técnicos e engenheiros trabalhando diretamente no chão de fábrica e dependeriam das formas de organização do trabalho; Melhorias em produtos e serviços estariam relacionadas com as interações de mercado e com firmas próximas (como subcontratadas e fornecedores). A P\&D formal seria decisiva às inovações radicais, conjuntamente com as relações externas da firma com outras instituições de um sistema técnico-científico profissional.

Após um enfoque teórico acentuado nos laboratórios de pesquisa e desenvolvimento como principal produtor de inovações, e principalmente o gasto monetário em P\&D, alguns autores começaram a perceber que apenas os investimentos em P\&D não eram suficientes para explicar as o desenvolvimento de inovações em algumas indústrias e regiões. Nesse sentido, Lundvall (1992) e Freeman (1995) foram os primeiros autores que trabalharam na construção do conceito de Sistema Nacional de Inovação (SNI). Um SNI pode ser definido como um conjunto de instituições, atores e mecanismos de um país, como os institutos de pesquisa, o sistema educacional, as firmas, as agências governamentais, a estrutura do sistema financeiro, as leis de propriedade intelectual e as universidades, que contribuem para a criação, avanço e difusão das inovações tecnológicas. A partir do conceito de SNI também é possível definir os Sistemas Regionais ou Locais de Inovação (SRI ou SLI), os quais seguem a mesma lógica, mas agora aplicado a nível regional ou local ao invés de nacional.

Edquist (2006) traz um bom resumo sobre a abordagem de sistemas de inovação e apresenta seus pontos fortes e fracos. Para o autor, a natureza do processo de inovação seria sistêmica visto que as firmas não inovam de forma isolada, mas em colaboração com outras organizações como firmas, universidades, escolas, institutos e agências governamentais. Além disso, o comportamento dessas organizações seria moldado pelas instituições, como leis, normas e regras, que criam os incentivos e obstáculos para a inovação. Segundo Edquist (2006) a abordagem sobre sistemas de inovação possui uma visão interdisciplinar e holística, colocando a inovação e os processos de aprendizagem no centro dos estudos. Para isso emprega perspectivas históricas e evolucionárias, enfatizando interdependência, não-linearidade e o papel das instituições. Nesse contexto, Gotardo, Staduto e Pontili (2017) realizaram um estudo com o propósito de investigar os fatores mais importantes para a localização industrial no Brasil utilizando como método modelo logit multinomial e multinível. Os autores encontraram como principal resultado a relevância da dos níveis de tecnologia em relação a localização industrial, assim como, economias de aglomeração.

Portanto, a inovação aparece como elemento central no desenvolvimento capitalista e aquelas regiões ou países que possuem sistemas de inovações eficientes tendem a apresentar melhores índices de desenvolvimento econômico. Dito isto, a seguir serão discutidas as questões da estrutura produtiva e das inovações.

\section{INOVAÇÃO, MUDANÇA TÉCNICA E TRAJETÓRIAS TECNOLÓGICAS}

Em seu trabalho seminal, Dosi (1982) realizou uma revisão crítica das teorias de mudança técnica e elaborou uma análise inovadora ao propor uma interpretação a respeito dos paradigmas tecnológicos e trajetórias tecnológicas. Para o autor o entendimento de tecnologia deve incluir a percepção de um conjunto limitado de alternativas tecnológicas possíveis e de desenvolvimentos especulativos futuros, incorporando a lógica de processo na análise. Outra importante percepção do autor é em relação ao que seria um paradigma tecnológico. Dosi (1982), influenciado pelo conceito Kuhniano de paradigma científico, define paradigma tecnológico como um 'modelo' e um 'padrão' de solução de determinados problemas tecnológicos, baseados em determinados princípios derivados das ciências naturais e em determinadas tecnologias materiais.

Essas elucidações são fundamentais para a compreensão da proposição de Dosi a respeito dos paradigmas tecnológicos, das trajetórias tecnológicas e consequentemente de como ocorre à relação entre as inovações e a economia. Essas percepções emergem, em grande medida, pela constatação das limitações das teorias vigentes para explicar a relação entre crescimento econômico e mudança

$\begin{array}{lllll}\text { Caminhos de Geografia } & \text { Uberlândia } & \text { v. 21, n. } 73 & \text { Mar/2020 } & \text { p. 243-252 Página } 245\end{array}$


econômica (progresso). Desse modo, Dosi (1982) argumenta que sua proposta de interpretação dos paradigmas tecnológicos e trajetórias tecnológicas não tem o objetivo de formular uma teoria geral da mudança técnica, mas, buscar trazer luz para questões como: Por que surgem alguns desenvolvimentos tecnológicos ao invés de outros? Existem regularidades no processo de geração de novas tecnologias e no progresso técnico? Existem padrões direcionais entre fatores econômicos, sociais, institucionais, científicos?

De um modo geral, o autor afirma que as teorias vigentes explicam o processo de mudanças técnicas por duas maneiras. As teorias nomeadas de "puxada pela demanda" e, de "impulsionada pela tecnologia". Na teoria puxada pela demanda os consumidores expressam suas preferências sobre as características dos produtos que desejam através de seus padrões de demanda; os produtores percebem as necessidades reveladas dos consumidores e iniciam o processo de inovação; as empresas bem-sucedidas, ao final, levarão ao mercado seus produtos novos e/ou melhorados.

Por outro lado, nas teorias nomeadas "impulsionada pela tecnologia" a mudança técnica, de um modo geral, ocorre quando existem pesquisas e desenvolvimento tecnológico de produtos e processos e seus resultados são colocados no mercado. Desse modo, o mercado desempenha um papel mais passivo. Essa explicação considera o processo inovativo mais "reativo" do que protagonista. Desse modo, a proposta de Dosi (1982 e 1988) explica a mudança técnica levando em consideração fatores como a) os paradigmas tecnológicos trazem a ideia de "progresso"; b) a tecnologia inclui a "percepção" de um conjunto limitado de alternativas tecnológicas possíveis; c) os paradigmas tecnológicos têm um poderoso efeito de exclusão como, por exemplo, grande desvantagem competitiva; d) a concorrência não ocorre apenas entre a tecnologia "nova" e a "antiga", que ela substitui, mas também com as alternativas das novas abordagens tecnológicas e d) outro poderoso critério de seleção nas economias capitalistas provavelmente será a capacidade de redução de custos da nova tecnologia.

Para o autor, o ambiente socioeconômico afeta o desenvolvimento tecnológico selecionando a "direção da mutação", ou seja, selecionando o paradigma tecnológico. Além disso, influencia ao fazer a seleção entre mutações, de forma darwinista, com o mercado atuando como um sistema de recompensas e punições. Nesse contexto, as novas tecnologias são selecionadas através de uma interação complexa entre: os fatores econômicos fundamentais, a busca de novas oportunidades e importantes fatores institucionais. Assim, o crescimento econômico e a transformação da economia envolvem uma realocação permanente dos recursos, bem como dos esforços de pesquisa entre diferentes setores. Ou seja, incorpora a ideia de processo dinâmico e o maior esforço inovativo será colocado nas áreas que oferecem crescimento relativo maior e oportunidades de lucro.

Nesse contexto, Asheim (1995) argumenta que a moderna teoria sobre inovação foi desenvolvida como uma crítica aos "modelos de inovação linear", pois estes seriam muito focados na inovação gerada a partir de conhecimentos formais e desenvolvida dentro dos laboratórios de P\&D. Existiria uma sobrevalorização da ideia que altos gastos em P\&D geram grandes quantidades de inovação e baixos gastos, baixas quantidades de inovação. Citando os trabalhos de Felsenstein (1994), Smith (1994), Lundvall (1992) e Camagni (1991), Asheim defende que o processo de inovação não envolve apenas pesquisa, mas, também, várias atividades relacionadas que compreendem um processo de interação entre firmas e seu ambiente. O processo de inovação, dessa forma, colhe frutos de uma maior interação social e um aprendizado interativo e depende de um processo de aprender fazendo, usando e falhando. Esse aprendizado interativo é socialmente enraizado e leva em consideração fatores institucionais e culturais. Assim, Lundvall \& Johnson (1994) cunharam o conceito de "learning economies" (economias de aprendizado) para definir uma economia capitalista moderna na qual o conhecimento é o principal recurso e o aprendizado o processo mais importante.

Asheim \& Gertler (2006, apud LAESTADIUS, 1998) distinguem as bases de conhecimento em dois tipos: conhecimento "analítico" e conhecimento "sintético". Uma base de conhecimento sintética ocorre em indústrias nas quais a inovação acontece principalmente pela aplicação e combinação do conhecimento já existente. $O$ processo inovativo transcorre com foco na eficiência e no desenvolvimento de novas soluções, sendo que o conhecimento é criado por processos de testes, experimentações e simulações. Normalmente, a inovação nessas indústrias surge para atender e solucionar problemas específicos. P\&D são menos importantes, usualmente, que em outros setores da economia. São exemplos desse tipo de base de conhecimento a indústria de maquinaria industrial, engenharia de planta e construção de navios (ASHEIM \& GERTLER, 2006).

Uma base de conhecimento analítica, por sua vez, é baseada em modelos formais, ciência codificada e processos racionais. Ela é um tipo de conhecimento que está presente em setores e indústrias em que o conhecimento científico é vital. Muitas das empresas que trabalham com esse tipo de base de

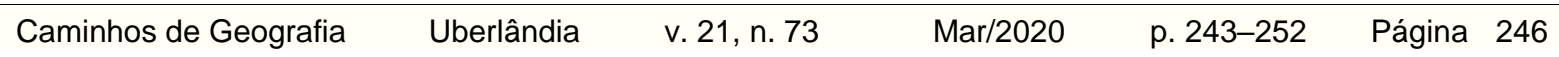


conhecimento têm seu próprio laboratório de P\&D ou trabalham em conjunto com centros de pesquisa e desenvolvimento. $O$ conhecimento analítico é mais codificado que sua forma sintética, pois sua geração é baseada no entendimento de princípios e métodos científicos e seus resultados são divulgados e registrados através de relatórios e podem dar origem a patentes, inclusive intelectuais. A inovação gerada pelo conhecimento analítico é capaz de proporcionar novos produtos e processos, com possibilidade de desenvolver uma inovação radical, algo que não é possível em indústrias que fazem uso do conhecimento sintético. Exemplos de indústria que utilizam essa base de conhecimento são a biotecnologia e a tecnologia da informação (ASHEIM \& GERTLER, 2006).

A inovação, estrutura produtiva e desenvolvimento econômico apresentam elementos de inter-relações. Um ponto central que surge no debate é justamente a inserção da dimensão territorial nessa discussão. A seguir serão debatidas as questões relacionadas às firmas, ao aprendizado e a importância da dimensão territorial nesse contexto de inovação.

\section{FIRMAS, APRENDIZADO E A IMPORTÂNCIA DA DIMENSÃO TERRITORIAL}

Maskell \& Malmberg (1999) afirmam que os fatores de produção e as capacidades locais se fazem presentes praticamente em todos os lugares em decorrência do processo de globalização, isto é, isso seria uma característica da própria globalização. No entanto, o que faz a diferença é o conhecimento não negociável e não-codificável devido à dificuldade de compartilhá-lo entre longas distâncias. Portanto, o conhecimento tácito se torna crucial e a proximidade espacial ganha destaque como fator de suma importância para explicar as diferenças da atividade inovadora entre regiões.

A transmissão de conhecimento tácito necessita de uma linguagem comum e de convenções e normas que possam ser compreendidas pelos envolvidos na troca. Essas propriedades ajudam a desenvolver confiança e destacam a importância da proximidade espacial e trazem a tona à importância da interação social.

A ideia que o conhecimento tácito é o que faz a diferença entre regiões que se destacam por sua capacidade de inovação é de certa forma contra intuitiva. No entanto, considere, por exemplo, que para o surgimento de uma inovação de processo ou produto é necessário uma série de testes e experimentos que, na sua maioria das vezes, acontecem por tentativas e erros. Quando a inovação acontece os resultados são documentados e divulgados internamente na empresa e externamente também, se assim a empresa desejar. Mas, note que apenas os resultados positivos são divulgados, são raras as vezes que os resultados de experimentos e testes que foram mal sucedidos são transmitidos seja para o público interno da empresa ou externo. No entanto, os experimentos falhos e os testes errados foram, provavelmente, de grande importância para a equipe de cientistas que desenvolveu a inovação. Suponha que a mesma equipe comece a trabalhar no desenvolvimento de uma nova inovação, essa equipe já possuirá uma bagagem de conhecimento devido aos seus sucessos e erros, enquanto que uma equipe de profissionais de uma região distante que apenas leu sobre o sucesso da inovação desenvolvida pela primeira equipe, não terá o conhecimento de todas tentativas erradas e frustradas que levaram ao surgimento da inovação. Isso faz com que a primeira equipe tenha mais experiência que a segunda equipe, mesmo que as duas tenham lido o mesmo relatório sobre o bem sucedido desenvolvimento de uma inovação.

Malerba (1992) propõe uma taxonomia na qual aponta seis tipos principais de processos de aprendizagem e como cada um está relacionado a diferentes fontes e tipos de conhecimento, sendo que muitos deles estão inter-relacionados. Os seis tipos seriam: a) learning by doing; b) learning by using; c) aprendizagem com os avanços na ciência e tecnologia; d) aprendizado com os transbordamentos (spillovers) interindustriais; e) aprendizado através da interação e f) aprendizado por pesquisa. O primeiro é caracterizado por ser interno à firma e relacionado a produção; O segundo também é interno à empresa e relacionado com a utilização de produtos, máquinas e insumos; O terceiro já é externo à firma e relacionada com a absorção de novos desenvolvimentos da ciência e tecnologia; O quarto também é externo à firma e relacionado com o que as concorrentes estão fazendo; $O$ quinto é igualmente externo à firma e diz respeito ao conhecimentos a montante e jusante do da interação com fornecedores e compradores ou até mesmo da cooperação com outras firmas; E por fim, o último, é interno à firma e frequentemente relacionado com atividades formais como P\&D, buscando gerar novos conhecimentos.

A partir destas discussões, Malerba (1992) argumenta que se pode esperar a relação seguinte (indireta) entre os processos de aprendizagem e as direções da mudança tecnológica incremental:

\begin{tabular}{llllll}
\hline Caminhos de Geografia & Uberlândia & v. 21, n. 73 & Mar/2020 & p. 243-252 & Página 247
\end{tabular}


i. Learning by doing e by using irá estimular a trajetória da elevação de produção-rendimento (yield);

ii. Aprendizado através da interação com os fornecedores estimulará trajetórias de melhorias de produção;

iii. Aprendizado através da interação com os fornecedores de insumos irá estimular trajetórias de mudança técnica periódica relacionada com as melhorias dos insumos;

iv. Aprendizado através da interação com os consumidores aprimorará a diferenciação horizontal do produto;

v. Aprendizado por pesquisa e P\&D estimulará a melhora em termos de diferenciação vertical do produto (qualidade e desempenho);

vi. As diferenças na base de conhecimento científico e tecnológico estarão por trás de toda a trajetória das mudanças técnicas incrementais.

Malerba (1992) chega a algumas conclusões ao analisar empiricamente os principais processos de aprendizagem das empresas e a relação entre os processos de aprendizagem e as trajetórias de mudança técnica incremental para os Estados Unidos. Primeiramente o autor conclui que o processo de aprendizagem está na raiz da mudança técnica. Além disso, conclui que as firmas podem ser vistas como organizações de aprendizado para aquisição, acumulação e criação de conhecimento nas quais vários processos de aprendizagem estão presentes indo muito além do tradicional learning by doing e apontando que os processos de aprendizagem estão intimamente relacionados com os rumos da mudança tecnológica incremental.

Portanto, a aprendizagem não é um bem livre, mas sim é uma atividade dispendiosa, focada e multidimensional (que diferencia as empresas). A aprendizagem por parte das empresas não se realiza em um vácuo. As fontes externas de conhecimento produtivo e tecnológico desempenham um papel importante na acumulação do estoque de conhecimento e do avanço técnico.

Segundo Queiroz (2006), no final do século dezenove e no início do século XX, o papel institucional no processo de inovação ganhou relevância. Nesse sentido, as firmas começaram a institucionalizar os processos de aprendizado e criaram estruturas especializadas como o departamento de P\&D. Em maior contexto, esses departamentos buscam inovações incrementais, no sentido de que desejam aprimoramentos de produtos e processos pré-existentes. Desse modo, ganha destaque essas interrelações entre as estruturas internas da firma, como o setor de pesquisa e desenvolvimento, por exemplo, e o contexto externo. Essas interações condicionam os direcionamentos dos processos de aprendizado e consequentemente da inovação em determinado espaço.

A dimensão territorial nesse contexto, pode ser definida como um recorte específico onde ocorrem processos produtivos, inovativos e cooperativos. Esses processos podem compreender municípios ou áreas de um município; conjunto de municípios; microrregião; conjunto de microrregiões, até mesmo países ou conjunto de países. A proximidade tende a auxiliar na vantagem competitiva produtiva. As regiões são importantes bases de coordenação econômica no nível mesorregional: "a região é cada vez mais uma localidade em que a inovação é produzida através de redes regionais, locais (clusters) e os efeitos de fertilização cruzada das instituições de pesquisa " (Lundvall e Borrás, 1999, p. 39).

Quando começamos a pensar a inovação como uma interação entre vários atores econômicos e sociais de uma região, entramos a literatura econômica dos sistemas regionais e locais de inovação (SRI ou SLI). Asheim (1998) e Asheim e Isaksen (2002) distinguem três tipos de SRIs. O primeiro tipo pode ser designado como Sistemas Regionais de Inovação (SRI) integrados regionalmente, na qual as empresas (principalmente as que utilizam conhecimento sintético) baseiam sua atividade inovativa principalmente em processos de aprendizagem estimulados pela proximidade geográfica, social e cultural, sem muita interação direta com as organizações de conhecimento. Os melhores exemplos são as obras de PMEs em distritos industriais.

Um segundo formato é o Sistema Regional de Inovação em rede. O sistema em rede é comumente considerado o tipo ideal de SRI: um conjunto regional de empresas cercado por uma região "apoiando" a infraestrutura institucional. Esse sistema é um resultado da intervenção política para aumentar a capacidade de inovação e colaboração regional e local. A trajetória "lock-in" (a incapacidade de se desviar de uma tecnologia estabelecida, mas ultrapassada) é um processo muito utilizado dentro dos clusters regionais nesse formato de SRIs.

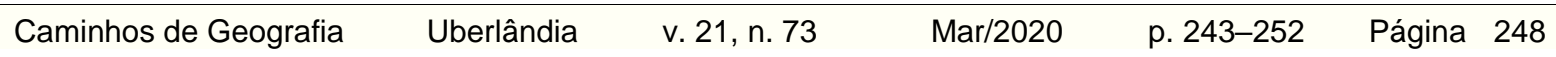


O terceiro modelo de SRI, o sistema de inovação nacional regionalizado, difere dos dois tipos anteriores devido a várias características. Parte da indústria e suas infraestruturas institucionais são mais integradas nas redes nacionais ou nos inter-sistemas nacionais de inovação - isto é, a atividade de inovação ocorre principalmente em cooperação com atores fora da região. Assim, isso representa um modelo de desenvolvimento em que seus atores e relacionamentos exógenos desempenham um papel maior.

Um exemplo de sistema de inovação nacional regionalizado é o agrupamento de laboratórios de P\&D em grandes empresas e/ou institutos de pesquisa governamentais nos "parques científicos-tecnológicos". Estes laboratórios podem estar localizados nas proximidades de universidades e faculdades técnicas, apesar de a evidência sugerir que os inquilinos dos parques científicos normalmente têm ligações limitadas com a indústria local (Asheim, 1995). Os parques científicos são, portanto, um exemplo típico de um meio inovador planejado composto por empresas de alto nível de recursos internos e competências, situados no local com fraca cooperação.

Pesquisadores produziram muitas evidências sobre a geografia altamente desigual da inovação. Uma abordagem importante foi construída ao medir os spillovers do conhecimento através do uso de indicadores como citações de patentes e a interação Universidade-Empresa. Nesse sentido, uma educação altamente qualificada faz com que trabalhadores na produção de inovações em indústrias analíticas procurem lugares que ofereçam as oportunidades de emprego mais atraentes. Isto é, esses trabalhadores são atraídos para aqueles lugares que oferecem uma forma de "buzz" baseado na carreira, e onde eles também podem encontrar uma massa crítica de pessoas que trabalham nas mesmas categorias profissionais ou similares. A dinâmica dos retornos crescentes gerará um poderoso círculo virtuoso no longo prazo de crescimento e dinamismo para esses setores intensivos em mão-de-obra altamente qualificada. Os lugares que oferecem uma alta qualidade de vida, além da carreira atraente, terão uma vantagem ainda maior na "batalha pelo talento". Esses trabalhadores altamente talentosos podem viver em muitos lugares, mas eles tendem a escolher viver nas cidades que ofereçam uma alta qualidade de vida, definida por um caráter social particular. Exemplos de regiões com essas características são o Vale do Silício e o distrito de Hollywood (STORPER; VENABLES, 2003 e FLORIDA, 2002).

Gertler (1995) investiga a importância do contexto espacial na relação entre produtores e usuários de maquinaria avançada para o sucesso da indústria de uma região. Ele realiza uma análise qualitativa exploratória, sendo que suas principais questões são se usuários e produtores precisam estar próximos para o desenvolvimento de uma relação de alta qualidade, se essa relação de alta qualidade é necessária para a implementação de alta tecnologia e se a noção de proximidade está relacionada à distância física ou a noções organizacionais e culturais. A pesquisa foi conduzida no Canadá. Os resultados mostram que a proximidade espacial é mais importante para as pequenas e médias empresas e para problemas relacionados a serviços e peças de reposição. Além disso, a distância física entre as empresas começa a ser mais relevante a partir de unidades de províncias, o que seria equivalente aos nossos estados, e que se torna extremamente importante quando se considera países. Fazendo uma analogia com o Brasil, pode-se especular que não é apenas a distância física que importa, mas também questões relacionadas com as diferentes culturas de cada estado, assim como, problemas relacionados a jurisdição, como diferenças de tributação, por exemplo. Especulações que quando consideradas para diferentes países apenas se ampliam.

Asheim \& Isaksen (1997) apresentam as características de duas aglomerações industriais da Noruega: o cluster de engenharia mecânica de Jaeren e o cluster de eletrônicos de Horten. A região de Jaeren é um centro de tecnologia robótica com alto viés exportador. Seu desenvolvimento começou após a Segunda Guerra Mundial com a criação da TESA (Cooperação Técnica) em 1957 pelas firmas locais em conjunto com escolas técnicas, seu objetivo é apoiar empresas médias exportadoras de maquinaria. A TESA, posteriormente, teve papel ativo na criação de um centro tecnológico na região cuja meta é assegurar um desenvolvimento tecnológico rápido e sustentado através, principalmente, de treinamento avançado de mão de obra.

Os autores defendem que a cooperação horizontal interfirmas na região é única. Essa cooperação tecnológica é apontada como a responsável pelo sucesso da região e foi desenvolvida a partir recursos internos e competências das firmas ao invés de instituições de pesquisa e desenvolvimento. Os valores comuns, como a ética de trabalho protestante, e os laços familiares próximos na comunidade também teriam contribuído no sucesso da região. Por outro lado, a região de Horten é sede de um complexo industrial de eletrônicos com inserção em um sistema de inovação nacional e internacional. A região possui 23 mil habitantes, o que equivale a uma cidade de tamanho médio na Noruega (segundo os

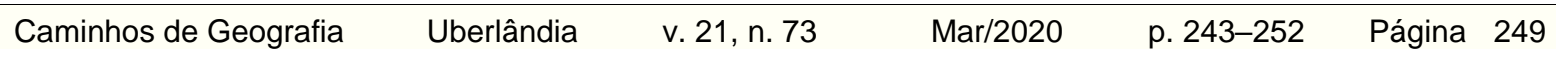


autores), e é detentora de $5 \%$ de todo o emprego industrial do país e um quociente locacional maior que 10 dentro dessa indústria. Existem grandes empresas que puxam o desenvolvimento da região e subcontratam parte do sistema produtivo para empresas menores. As maiores empresas possuem seus próprios laboratórios de P\&D dentro de seus estabelecimentos e o desenvolvimento de novos produtos é financiado, muitas vezes, pelo Conselho de Pesquisa da Noruega. Existe pouca cooperação horizontal na região e as relações entre grandes e pequenas empresas envolvem relações de poder assimétricas, o que cria a necessidade de grande flexibilidade por parte das pequenas empresas.

Ao final de sua pesquisa, Asheim \& Isaksen (1997) concluem que políticas de inovação descentralizadas são necessárias para aproveitar melhor as especificidades territoriais, pois a inovação ocorre de formas diversas em cada região, dependendo da estrutura da indústria e da firma e das características sociais e culturais de cada uma delas.

\section{CONSIDERAÇÕES FINAIS}

O objetivo deste artigo foi construir um debate de teorias que relacionam a Geografia, Inovação e o Desenvolvimento Econômico. Sobretudo, argumentando que a proximidade geográfica é a chave para a produção efetiva e transmissão partilhada do conhecimento tácito, reforçando a importância dos aglomerados inovadores, distritos e regiões.

Por meio da literatura apresentada e de alguns estudos empíricos relatados, pode-se argumentar que o impulso fundamental do capitalismo advém dos novos bens de consumo, métodos de produção ou transporte, dos novos mercados, das novas formas de organização industrial, ou seja, a inovação ganha destaque central neste modo de produção. Um ponto essencial que surge no debate é justamente a inserção da dimensão territorial nessa análise. Essa inserção ocorre, dentre outros motivos, pela existência e importância do conhecimento tácito no processo inovador.

Ademais, introduzimos a discussão acerca dos sistemas regionais de inovação, ressaltando os elementos e caráter sistêmico que compõem uma parte chave de uma região distinta institucionalmente. Também foi evidenciado a variedade de diferentes tipos de sistemas que podem ser identificados. Foi possível observar que embora haja significante variação no desempenho econômico em diferentes regiões dentro do mesmo sistema regional, as características dos sistemas de inovação regional bem-sucedidos sob o mesmo regime nacional exibirão certas consistências de caso a caso.

Uma economia altamente competitiva e globalizada parece ser cada vez mais difícil executar a reprodução e o crescimento do processo de aprendizagem, que dependem principalmente do incremento de melhorias em produtos e processos e não apenas de novos produtos. A ideia fundamental dos sistemas regionais de inovação é que a promoção de novos processos de aprendizagem concentrados pode melhorar a inovação e vantagem competitiva das economias regionais. O grande questionamento que se faz é como a capacidade dos sistemas regionais de inovação para atualizar as bases do conhecimento das empresas se desenvolverá ao longo do tempo.

Desse modo, este artigo destaca a importância da análise geográfica e da dimensão territorial em estudos que tenham como propósito compreender o processo inovativo, desenvolvimento econômico e consequentemente a economia globalizada contemporânea.

\section{REFERÊNCIAS}

ASHEIM, B. Industrial districts as "learning regions". A condition for prosperity? STEP report R03/1995, 1995.

ASHEIM, B.; ISAKSEN, A. Location, agglomeration and innovation: Towards regional innovation systems in Norway? European Planning Studies, v. 5, n. 3, 1997. https://doi.org/10.1080/09654319708720402

ASHEIM, B; GERTLER, M.S. The Geography of Innovation - Regional Innovation Systems.In: FAGERGERG, J.; MOWERY, D.; NELSON, R. (Eds). The Oxford Handbook of Innovation. Oxford: Oxford University Press, p. 181-208, 2006. https://doi.org/10.1093/oxfordhb/9780199286805.003.0011

ASHEIM, Bjørn T.; GERTLER, Meric S. The geography of innovation: regional innovation systems. In: The Oxford handbook of innovation. 2005. https://doi.org/10.1093/oxfordhb/9780199286805.003.0011

$\begin{array}{llllll}\text { Caminhos de Geografia } & \text { Uberlândia } & \text { v. 21, n. } 73 & \text { Mar/2020 } & \text { p. 243-252 Página } 250\end{array}$


CAMAGNI, R. Introduction: from the local "milieu" to innovation through cooperation networks, in Camagni, R. (ed.), Innovation networks: spatial perspectives. Belhaven Press, London, 1-9. 1991.

DOSI, G. Technological paradigms and technological trajectories: a suggested interpretation of the determinants and directions of technical change. Research policy, v. 11, n. 3, p. 147-162, 1982.

DOSI, G. The Nature of the Innovative Process. in G. Dosi et al. (eds.), Technical Change and Economic Theory, London: Pinter, 221-38. 1988. https://doi.org/10.1016/0048-7333(82)90016-6

EDQUIST, C. Systems of Innovation: perspectives and challenges. In: FAGERGERG, J.; MOWERY, D.; NELSON, R. (Eds). The Oxford Handbook of Innovation. Oxford: Oxford University Press, p. 181-208, 2006. https://doi.org/10.1093/oxfordhb/9780199286805.003.0007

FELSENSTEIN, D. Book review essay (on Massey, D. et al, High tech fantasies, London 1992). Economic Geography, 70, 1, 72-75. 1994. https://doi.org/10.2307/143579

FLORIDA, R. The Economic Geography of Talent. Annals of the Association of American Geographers 92: 743-55. 2002. https://doi.org/10.1111/1467-8306.00314

FREEMAN, C. The National Innovation Systems in historical perspective. Cambridge Journal of Economics, vol. 19, no. 1, 1995.

GERTLER, M.S. Best practice? Geography, learning and the institutional limits to strong convergence. Journal of Economic Geography, 1, p. 5-26, 2001. https://doi.org/10.1093/jeg/1.1.5

GERTLER, M.S. Tacit Knowledge and the economic geography of context, or The undefinable tacitness of being (there). Journal of Economic Geography, 3, p. 75-99, 2003. https://doi.org/10.1093/jeg/3.1.75

GOTARDO, D. M.; STADUTO, J. A. R. . POLOS E ÁREAS DE INFLUÊNCIA: UMA PROPOSTA DE REGIONALIZAÇÃO ECONÔMICA PARA O ESTADO DO PARANÁ. REVISTA BRASILEIRA DE ESTUDOS URBANOS E REGIONAIS (ANPUR), v. 19, p. 106-124, 2016. https://doi.org/10.22296/2317-1529.2017v19n1p106

GOTARDO, D. M. ; STADUTO, J. A. R. ; PONTILI, R. M. . DETERMINANTES DA LOCALIZAÇÃO INDUSTRIAL: UMA ANÁLISE PARA AS MESORRGEGIÔES BRASILEIRAS. In: I Latin American and Caribbean Regional Science Association Congress XV Encontro Nacional da Associação Brasileira de Estudos Regionais e Urbanos, 2017, São Paulo. ANAIS XV ENABER / I LACRSA, 2017.

LAESTADIUS, S. 'Technology Level, Knowledge Formation and Industrial Competence in Paper Manufacturing. In G. Eliasson et al. (eds.), Microfoundations of Economic Growth. A Schumpeterian Perspective, Ann Arbor: University of Michigan Press, 212-26. 1998.

LUNDVALL, B.-Å. National Innovation Systems: Towards a Theory of Innovation and Interactive Learning. Londres: Pinter Publishers, 1992. https://doi.org/10.1080/13662719400000002

LUNDVALL, B.-A.; JOHNSON, A. B. The learning economy. Journal of Industry Studies, 1,2, 23-42. 1994.

LUNDVALL, B.; BORRÁS, S. The Globalising Learning Economy: Implications for Innovation Policy. DGXII-TSER, The European Commission. 1999.

MALERBA, Franco. Learning by firms and incremental technical change. The economic journal, v. 102 , n. 413 , p. 845-859, 1992. https://doi.org/10.2307/2234581

MASKELL, P.; MALMBERG, A. Localised Learning and Industrial Competitiveness, Cambridge Journal of Economics, 23: 167-86. 1999. https://doi.org/10.1093/cje/23.2.167

NELSON, R.; WINTER, S. An evolutionary theory of economic change. Belknap Press, Cambridge. 1982.

POSSAS, Mario Luiz. Em direção a um paradigma microdinâmico: a abordagem neoschumpeteriana. Ensaios sobre economia política moderna: teoria e história do pensamento econômico. São Paulo: Marco Zero, p. 157-177, 1989.

QUEIROZ, Sérgio. Aprendizado tecnológico. Economia da inovação tecnológica. São Paulo: Editora da Ordem dos economistas do Brasil, 2006. 
SCHUMPETER, J. A. A Teoria do Desenvolvimento Econômico. São Paulo: Abril Cultural, 1982/1911.

SMITH, K. New directions in research and technology policy: Identifying the key issues. STEPreport, No. 1, The STEP-group, Oslo. 1994.

STORPER, M.; VENABLES, A. J. Buzz: The Economic Force of the City. Paper presented at the DRUID Summer Conference 2003 on "Creating, sharing and transferring knowledge: the role of geography, institutions and organizations" Elsinore, Denmark. 2003.

Recebido em: 09/06/2019

Aceito para publicação em: 22/11/2019 